operated. On an industrial scale, filter cloths with enzyme action can be mounted in a plate-and-frame filter press; in the laboratory scale, small pieces of active paper can be used by mounting them in a filter holder (Gelman Instrument Co., Michigan). In this way, good flow rates can be obtained without plugging or channelling, and the bed size can readily be altered by adding or removing sheets. By using several sheets with different enzymes attached, it is possible to build a "multi-enzyme" reactor.

We thank Mr R. M. Patel for technical assistance This work was supported by a grant from the Science Research Council and is the subject of patent applications, the rights of which have been assigned to NRDC.
G. KAY
M. D. LILLY
A. K. SharP
R. J. H. WILSON

Biochemical Engineering Section,

Department of Chemical Engineering,

University College, London.

Received January 18, 1968.

${ }^{1}$ Hornby, W. E., Lilly, M. D., and Crook, E. M., Biochem. J. (in the press). ${ }^{2}$ Lilly, M. D., and Sharp, A. K., The Chemical Engineer (in the press).

${ }^{3}$ Goldstein, L., Levin, Y., and Katchalski, E., Biochemistry, 3, 1913 (1964).

- Silman, H. I., and Katchalski, E., Ann. Rev. Biochem., 35, 873 (1966).

${ }^{5}$ Kay, G., and Crook, E. M., Nature, 216, 514 (1967).

\section{De-esterification using Ion Exchangers}

IoN exchangers, as the name implies, are used principally for the removal of unwanted ions, but they have also been used for ionic catalysis. In particular, cation exchangers in the $\mathrm{H}^{+}$form have been employed to catalyse reactions requiring $\mathrm{H}^{+}$in which the product is required free from acid.

The hydrolysis of esters can be catalysed by acids or bases, but the latter are more effective. Esters of different types of alcohol (primary, secondary, hemiacetal) have different stability to base-catalysed hydrolysis. This property was employed in the selective hydrolysis of peracetylated sugars with acetonic potassium hydroxide to give compounds which are esterified at the primary hydroxyl groups only1-3. The sugar products of these reactions were accompanied by potassium acetate from which they had to be separated.

This communication describes the use of anion exchange columns to accomplish two tasks: first, to catalyse the hydrolysis of esters and, second, to absorb the acid so formed. Peracetylated sugars and sugar alcohols were used as starting materials. Preliminary experiments indicated that weak anion exchangers such as 'Dowex 3 ' or 'Duolite $A 6$ ' were not effective and so 'Dowex 1' was used. A column of this material effectively hydrolyses $\beta$-glucose pentacetate or $\beta$-galactose pentacetate to the free sugar, or sorbitol hexacetate to the alditol at room temperature. If, however, the temperature is below $5^{\circ} \mathrm{C}$, then the deacetylation is not completed. Thus 'Dowex I' $\times 1$ 50-100 mesh (400 g) was equilibrated with normal sodium hydroxide, washed three times with water and twice with actone : water $1: 1$ and placed in a cooled Liebig condenser measuring $200 \times 2 \mathrm{~cm}$ and washed with acetone-water until the $p \mathrm{H}$ of the eluant was below 8 . Ten grams of $\beta$-glucose pentacetate or $\beta$-galactose pent. acetate in $20 \mathrm{ml}$. acetone was then applied to the column, which was maintained below $5^{\circ} \mathrm{C}$. The column was eluted with acetone-water. The first $650 \mathrm{ml}$. of eluate contained the products, which were a mixture of several acetates of the sugars, with 6-O-acetyl glucose or 6-O-acetyl galactose. respectively, predominating. These were crystallized and identified. When sorbitol hexacetate was applied, thin layer chromatography of the eluate (silica-gel $D 5$ from Camag developed with chloroform : methanol, $88: 12$, and made visible with iodine), showed the product to be a mixture of acetates. Of these, the 1,6-di-O-acetyl sorbitol predominated. Also present were monoacetyl sorbitol, esterified at a primary alcoholic group, and triacetyl sorbitol (esterified at C1, C6 and C2 or possibly C5) and some tetraacetyl sorbitol.

At both room temperature and in the cold, the column absorbed the acetate that was formed. Attempts to use the column for a third task, namely, that of separating the sugar-ester products from each other, were unsuccessful.

Preliminary experiments indicate that the method of ion exchange de-esterification would be widely applied, not only for sugar esters but also for lipids. Thus glyceryl tripropionate was hydrolysed to a mixture of glycerol and monoglycerate. A paper chromatogram of the latter reacted slowly with alkaline permanganate-periodate spray (for glycols), and weakly with the alternate periodate- $p$-anisidine spray ${ }^{5}$ but strongly with alkaline hydroxylamine-acidic ferric chloride $\operatorname{spray}^{B}$ (for esters). Quantitative periodate oxidation confirmed that it was a mixture of $\alpha$ - and $\beta$-monopropionin. The mixture could have arisen as a result of acyl migration ${ }^{7}$.

Polyacetylated oligosaccharides can also be deacetylated in this fashion, for the 'Dowex l' column does not affect most glycosidic linkages. Even the relatively labile $\beta$ fructofuranoside linkage is not hydrolysed, and, on deacetylation, sucrose octacetate gave sucrose admixed with sucrose monoacetate. On the other hand, in suitable conditions, 'Dowex 50' and other cation exchangers hydrolysed glycoside bonds but not acetyl bonds.

I thank Professor J. Leibowitz for his advice and $\mathrm{Mr}$ M. Levanon for his technical assistance.

Y. Z. FrohweIN

Laboratory of Organic and Biological Chemistry, Hebrew University,

Jerusalem.

Received December 21, 1967.

Frohwein, Y. Z, and Leibowitz, J., Nature, 186, 153 (1960).

Frohwein, Y. Z, Nature, 196, 775 (1962).

${ }^{3}$ Frohwein, Y. Z., and Leibowitz, J., Bull. Res. Coun., Israel, 11A, 330 (1963).

'Lemieux, R. U., and Bauer, H. F., Anal. Chem., 26, 920 (1954).

"Bragg, P. D., and Hough, L., J. Chem. Soc., 4050 (1958).

${ }^{6}$ Frohwein, Y. Z., Israel J. Chem., 2, 57 (1964).

'Borgstrom, B., Acta Physiol. Scand., 30, 231 (1954).

\section{RADIOBIOLOGY}

\section{Attenuation in the Chest Wall of $20 \mathrm{keV} X$-rays from an Inhaled Radioactive Aerosol}

EXTERNAL counting of the low intensity $L$ uranium X-rays (energies $13 \cdot 6,16 \cdot 9$ and $20 \cdot 2 \mathrm{keV}$ ) is a promising technique for the estimation of insoluble compounds of plutonium in the human lung. These low energy radiations are, however, very easily absorbed in the tissues of the chest wall; the half-value thickness (HVT) is about $0.7 \mathrm{~cm}$ of soft tissue and only $0.03 \mathrm{~cm}$ of bone. The effects of variations in body build are clearly important and difficult to assess, and calibration of the counting equipment is a severe problem. One approach has been the use of a phantom ${ }^{1}$ or cadaver ${ }^{2}$. In neither of these cases is there any certainty that the radiation from plutonium inserted into the chest cavity will undergo the same attenuation by surrounding tissues as in vivo.

There are now techniques for the preparation of monodisperse aerosols of polystyrene particles, labelled with radio-nuclides, and suitable for inhalation by volunteers ${ }^{3}$. This has made it possible to study the plutonium calibration problem in vivo, using radio-nuclides which decay by 